\title{
Adaptive Walking assistance based on Human-Orthosis Interaction*
}

\author{
Vijaykumar Rajasekaran, Joan Aranda, Member, IEEE, and Alicia Casals, Senior Member, IEEE
}

\begin{abstract}
An assistive rehabilitation strategy for a lowerlimb wearable robot is proposed and evaluated. The control strategy monitors the human-orthosis interaction torques and modifies the orthosis operation mode depending on its evolution with respect to a normal gait pattern. The control algorithm relies on the adaptation of the joints stiffness in function of these interaction torques and to the deviation from the desired trajectory. A walking pattern, an average of recorded gaits, is used as reference input. The human-orthosis interaction torques are used to define the time instant when robot assistance is needed and its degree. The objective of this work is to demonstrate the feasibility of ensuring a dynamic stability by means of an efficient real-time stiffness adaptation for multiple joints and simultaneously maintaining their synchronization. The algorithm has been tested with five healthy subjects showing its efficient behavior in maintaining the equilibrium while walking in presence of external forces. The work is performed as a preliminary study to assist patients suffering from Spinal cord injury and Stroke.
\end{abstract}

Keywords - Exoskeleton, Gait assistance, Human-orthosis interaction, Impedance control, Wearable robot

\section{INTRODUCTION}

Assistive walking using an exoskeleton constitutes a major step forward in rehabilitation robotics. The assist-asneeded concept determines the level of assistance that the robot provides to the patient. Walking based on completely assisted robot therapies induce slacking to the patient, so it is necessary to provide a personalized assistance dynamically adapted to the changing patient's needs. The level of assistance varies with regard to the patient and the therapy, which involves the knowledge of assistance to be perceived. In classic control, the assistance can be either position or force based or a combination of both. These kinds of assistance can be improved with other factors such as the hardware structure, control strategies, combination of assistive devices etc. One of the widely used approaches to detect and evaluate the need of assistance is by evaluating the position errors. However, the use of a predefined trajectory pattern, without other inputs, imposes a complete assistance without considering the status and progress of the user. It is necessary to measure the human-orthosis

*This research is supported by HYPER project (Hybrid Neuroprosthetic and Neurorobotic devices for functional compensation and rehabilitation of motor disorders), grant CSD2009-00067 CONSOLIDER INGENIO 2010 from MINECO (Spanish Ministry for Science and Education)).

Vijaykumar Rajasekaran is associated with the Department of Automation and control, Universitat Politècnica de Catalunya, Barcelona-Tech, Spain. (corresponding author email: rajasekaran.vijaykumar@gmail.com)

Joan Aranda and Alicia Casals are associated with the Department of Automation and control, Universitat Politècnica de Catalunya, BarcelonaTech and Institute for Bioengineering of Catalonia, Barcelona, Spain. interaction torques to design a hybrid force-position control.

An adequate degree of assistance in robotic rehabilitation can be achieved using an effective control strategy [1] such as impedance or adaptive control which acts based on the subject's performance. These control strategies operate under the principle of assistance-as-needed, in which assistive forces increase as the participant deviates from the desired trajectory. This type of user-performance based adaptive control can be modeled as a spring-damping coupled system. Several human centered strategies, such as patient cooperative and support motor function assessment, oriented to the development of robot behaviors have been widely studied [2]. These strategies allow the development of patient driven behaviors that rely on the active force contributed by the patient, to achieve the desired trajectories. This kind of human centered control has proved to be efficient in robot-aided treadmill training, in systems like Lokomat and LOPES, which are provided with an adapted bodyweight support system [3,4]. Each joint movement in LOPES is supported by two motor actuations which facilitate the adequate compliant assistance by mimicking a muscle like behavior. On the contrary, HAL (Hybrid Assistive limb), a light weight powered exoskeleton suit, is efficient in adapting to the user movements by sensing the muscle synergies. While LOPES and Lokomat use interaction forces between human and orthosis, HAL uses EMG signals to measure the forces which are used to determine the joint stiffness to be applied [5].

In wearable robots or exoskeletons, the dynamic stability in walking plays an essential role in the assistance to be provided, which relies on the ground reaction forces acting on them. Several commercially available exoskeletons have succeeded in providing dynamic stability, such as BLEEX [6] Ekso (earlier eLegs) [7], Rex (Rex Bionics) and Re-Walk [8]. These exoskeletons have proven to be efficient in providing assistance on a passive range of motion, but using complex systems.

The objective of this work is to advance a step forward in the implementation of an impedance based approach for walking using a wearable robot, without treadmill training and body weight support. The absence of weight compensation carries with it the challenging task of maintaining the equilibrium in presence of ground reaction forces. The goal is to develop an efficient control model for a low-cost wearable robot and to validate the assistive behavior of the robot for patients with neurological disorders. A hybrid position and interaction torques based control strategy is presented to continuously adapt the user movements to the desired gait pattern in real time. This real time adaptation also ensures the synchronization among the joint trajectories to maintain the dynamic stability. 


\section{GAIT ADAPTATION}

The mathematical model for the dynamic analysis of an exoskeleton includes the human-orthosis interaction torques, as given in equation 1 .

$$
M_{\text {ort }}(q) \ddot{q}+C_{\text {ort }}(q, \dot{q})+G_{\text {ort }}(q)=\tau_{a}+\tau_{\text {pat }}+\tau_{d}
$$

where $q, \dot{q}, \ddot{q}$ are the vectors of joint positions, velocities and accelerations. $M_{\text {ort }}(q)$ is the inertia matrix, $C_{\text {ort }}(q, \dot{q})$ is the centrifugal and Coriolis vector and $G_{\text {ort }}(q)$ represents the gravitational torques. $\tau_{a}$ and $\tau_{\text {pat }}$ are the orthosis and patient torques respectively and $\tau_{d}$ correspond to the external disturbances acting on the subject. These actuator and patient torques are influenced by the human-orthosis interaction, while the external disturbances can be due to any assistive or external sources which can affect the dynamic stability of the robot.

In the present work, for each joint, the actuator works in collaboration with the patient. The actuator torque can be modified by varying the joint stiffness parameter, which invariably modifies the corresponding joint trajectory. Impedance control can be implemented using either a conventional nonlinear control law or an inner position control loop covered by force compensation, as an outer loop. In this case, the exoskeleton uses the second method, since the usage of a non-linear law is always associated with stability problems [9]. Force compensation is achieved by varying the stiffness parameter of the joint. The joint stiffness variation modulates the torque that determines the degree of control transferred from the orthosis to the human or vice versa. Such an impedance control scheme has been widely applied due to its compliant behavior, which results in a natural and realistic walking pattern and a more natural interaction between patient and orthosis. This impedance control can be determined by equation (2)

$$
F=M a+C v+K \cdot\left(\theta_{\text {ref }}-\theta_{\text {act }}\right)
$$

where, $\theta_{\text {ref }}$ and $\theta_{\text {act }}$ are the reference and actual joint positions respectively, $\mathrm{K}$ is the stiffness parameter of the joint and $\mathrm{F}$ represents the force to be applied on the joint. $\mathrm{M}$ represents the mass, $\mathrm{C}$ is the damping constant and $a$ and $v$ represent the acceleration and velocity of the robot. Since the input rate to the system is maintained constant, the velocity of the orthosis is not modified by the user and thus does not induce any significant effect on the applied force. Hence the force equation, influenced by the position error, is modified as

$$
F=K\left(\theta_{\text {ref }}-\theta_{\text {act }}\right)
$$

The value $\mathrm{K}$ can be determined based on the performance of the user and the level of assistance to be exerted by the orthosis.

$$
\begin{aligned}
& K_{t+1}=K_{t} \pm \Delta K \\
& \Delta K=\left|\left(\frac{\theta_{r e f}-\theta_{a c t}}{s * \tau_{\text {int }}}\right)\right|
\end{aligned}
$$

where, $\tau_{\text {int }}$ is the human-orthosis interaction torques and $s$ is a confidence factor $[0,1]$ which is used to determine the stiffness to be applied at time $t+1$. The confidence factor is a variable parameter which shall be defined by the therapist in function of the capabilities of the patient. This confidence factor can be varied according to the progress of the user [0, 1], in order to modify the time instant at which assistance is to be initiated. A low confidence factor means that the assistance should be provided partially or completely to the procedure and a higher confidence factor indicates that the subject is capable of walking without or with little assistance.

A variation of the $K$ value results in a change in the force acting at the joint level what is perceived as assistance or resistance by the patient. Thus, the $\mathrm{K}$ value is incremented or decremented as a function of the evolution of the patient. This stiffness value is computed from the analysis of interaction torques, thus determining the impedance to be exerted by the exoskeleton.

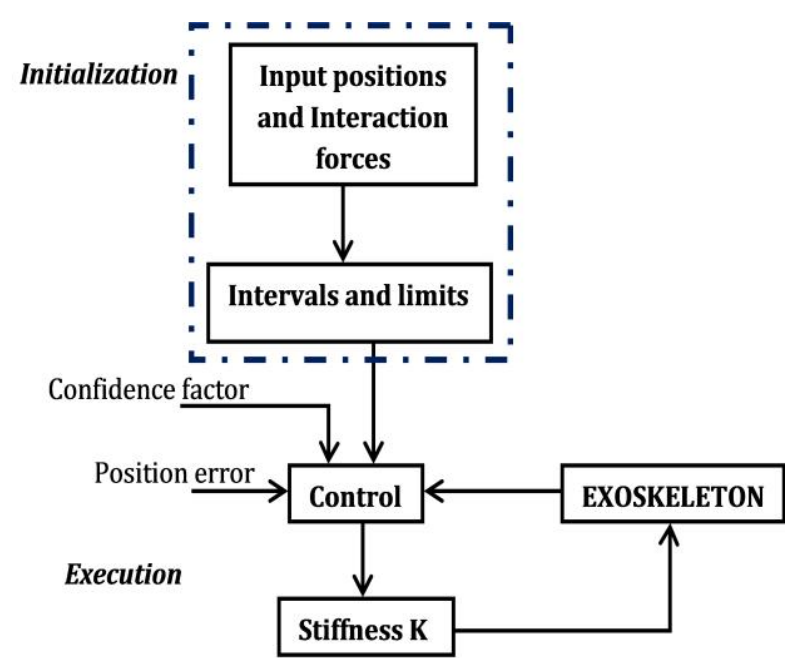

Figure 1. Schematic representation of the adaptive control strategy

\section{Stiffness variation:}

In this work the variation of stiffness is determined by both, position errors and human-orthosis interaction torques. The concept of assistance as needed is implemented by varying the joints impedance. The stiffness variation module, as shown in fig 1, is responsible for incrementing, decrementing or maintaining the stiffness parameter of each joint. Within a given range of trajectory errors, stiffness is computed from position errors, but when the defined error thresholds are surpassed, stiffness should be modified according to the measured interaction torques. This condition takes place in general with the presence of external perturbations. The following are the parameters involved in defining this stiffness variation:

$\theta_{e}$ - Position error (deg)

$\theta_{e} T h_{u p}-$ Upper threshold of position error (deg)

$\theta_{e} T h_{l o}-$ Lower threshold of position error (deg)

$\tau_{\text {int }}$ - Human-orthosis interaction torques (Nm)

$\tau_{\text {int }} T h_{l o}$ - Lower threshold of interaction torques (Nm)

$\tau_{\text {int }} T h_{u p}$ - Upper threshold of interaction torques $(\mathrm{Nm})$

$\Delta K$ - Stiffness variation $(\mathrm{N} / \mathrm{m})$ 


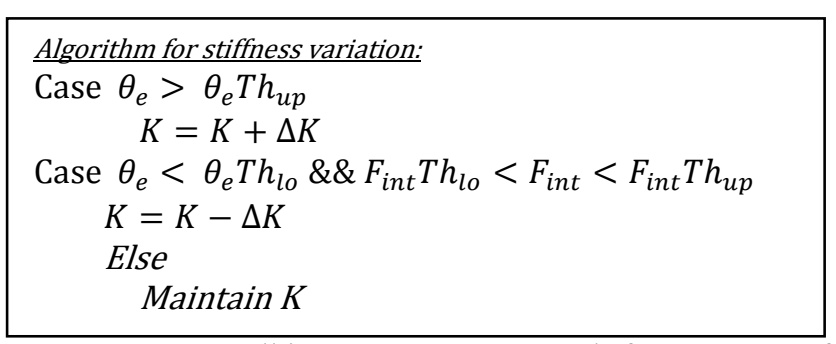

An average walking pattern generated from a set of recorded walking patterns of healthy individuals is used as the reference input. Based on this pattern and the patient contribution, the stiffness $\mathrm{K}$ establishes the operating mode at each joint, assistive or resistive. In a first set of trials, for each subject the maximum interaction torques are obtained by applying a low stiffness value at each joint. From these maxima, we extract the upper and lower thresholds of interaction torques to dynamically define the operation mode. These thresholds are obtained by multiplying these maxima interaction torques by the confidence factor $(s)$, where the upper and lower threshold values indicate the maxima of the positive and negative interaction torques respectively. The evolution of the threshold limits of the interaction torques and the position errors at a given time interval is shown in fig 2. A position error beyond the threshold limits means that the subject is not able to achieve the desired trajectory. Two situations might prevent reaching this trajectory,

1- The subject applies his maximum effort and tries to move faster than desired which means that the specific joint of the exoskeleton has to apply a resistive actuation, to ensure synchronization in walking.

\section{2- The patient is progressing slowly and needs assistance.}

In the first case, it is necessary to provide some corrective actuation, by increasing the stiffness of the joint such that the user does not lose his/her postural stability. In the second case, the stiffness is maintained or decreased in the corresponding joint, so as to allow the subject to keep walking at his/her own pace.

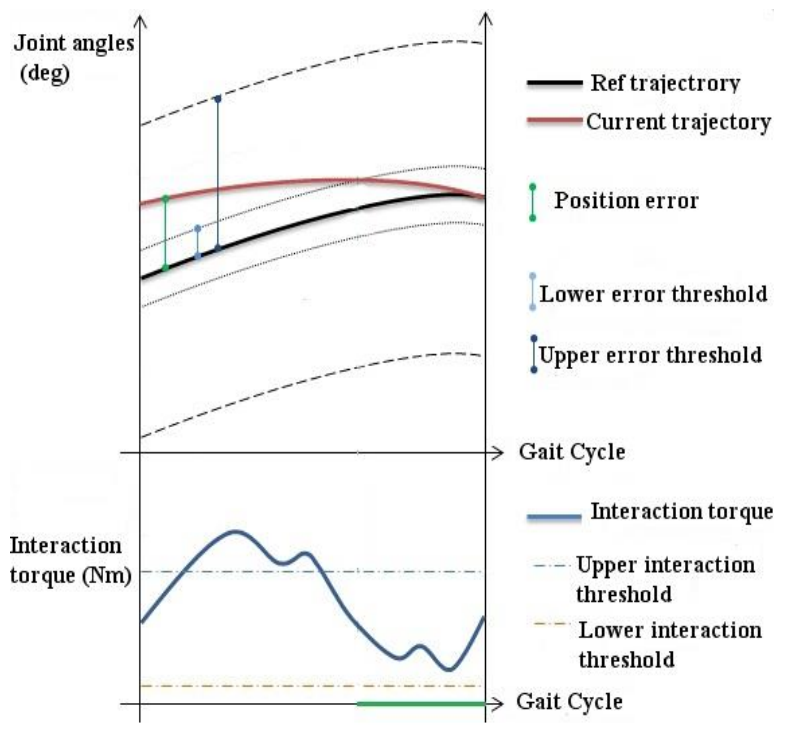

Figure 2. Relation between position error and interaction force

\section{EXPERIMENTAL PROCEDURE}

The proposed adaptive strategy is based on the position error and human-orthosis interaction torques. Hence, the experimentation consists of two phases: initialization and execution. The initialization phase involves monitoring the interaction torques and joint positions with no-assistance provided by the orthosis in order to be able to define $s$. In the execution phase, these interaction torques are limited with the confidence factor to determine the time instants of stiffness variation. Both these phases are performed using a lower-limb exoskeleton.

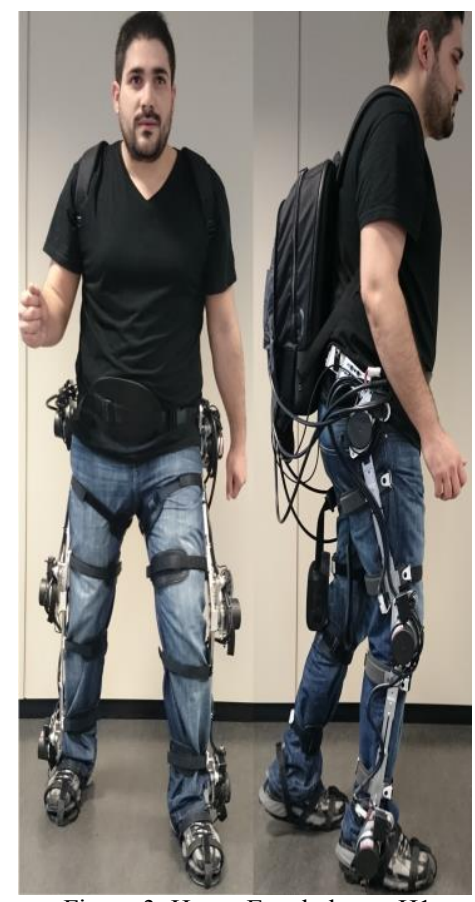

Figure 3. Hyper Exoskeleton, H1

\section{A. Exoskeleton:}

The proposed adaptive control strategy based on the human-orthosis interaction for assistive walking is evaluated using a lower limb exoskeleton, H1. H1 is a 6 DoF (degree of freedom) wearable lower limb orthosis with an anthropomorphic configuration to assist individuals with SCI or Stroke. The exoskeleton, shown in fig 3, has been built within the framework of the Hyper* project. H1 has three joints for each leg: hip, knee and ankle, each joint is powered by a DC motor coupled with a harmonic drive gear. The exoskeleton is equipped with potentiometers and strain gauges to measure the joint angles and human-orthosis interaction torques on the links respectively. Detailed descriptions about the exoskeleton structure and communication parameters involved are detailed in [10]. The variable stiffness control ensures a safe therapeutic experience. The exoskeleton permits a stiffness value within the range of $1-100 \mathrm{~N} / \mathrm{m}$. A low stiffness value $(<10 \mathrm{~N} / \mathrm{m})$ will not cause any significant effect on the user's behavior. Similarly a high stiffness value $(>80 \mathrm{~N} / \mathrm{m})$ will provide a completely assisted movement, with few or no input from the user [11]. The initial stiffness value must be defined 
taking into account the user's capability and the degree of assistance to be applied by the orthosis.

\section{B. Experimentation:}

Initially the walking pattern is tested on subjects applying a low stiffness value $(20 \mathrm{~N} / \mathrm{m})$, which is used to obtain the pattern of interaction torques and to adapt themselves to the orthosis. The evolution of these interaction torques are used to determine the initiation of stiffness variation, determining the adaptive behavior to be exerted by changing the stiffness. This variable stiffness results in either an assistive or resistive behavior, based on the movement. The joint stiffness values vary according to the position error and the trend of the interaction torques produced. The confidence factor is used to determine the time instants of actuating the stiffness function of the joint gradually. This assists in achieving a smooth performance without affecting the joint trajectory. The gradual increase or decrease of the stiffness value indicates the difference in interaction torques.

Table I. Initial parameters of this analysis

\begin{tabular}{|c|c|}
\hline Gait velocity & 5 seconds/cycle \\
\hline Initial stiffness value & $50 \mathrm{~N} / \mathrm{m} / \mathrm{deg}$ \\
\hline Upper Position error & $5^{\circ}$ \\
\hline lower position error & $1^{\circ}$ \\
\hline Stiffness increment & $1 \mathrm{~N} / \mathrm{m} / \mathrm{deg}$ \\
\hline Confidence factor & 0,9 \\
\hline
\end{tabular}

The setup includes a recorded gait pattern performed by healthy users and optimized after some repetitions of gait cycles. The values of stiffness and confidence factor are defined based on the subject's health condition. Table 1 lists the initial parameters used in this experimental procedure. Since the strategy is tested with healthy individuals, the initial stiffness and confidence factor are assumed to be 50 $\mathrm{N} / \mathrm{m}^{2}$ and 0.9 respectively. High interaction torques are found in healthy subjects, so a higher confidence factor is needed to define their thresholds. In case of SCI patients, we consider that this value must be between $0.5-0.7$, such that the assistance and resistance are provided whenever needed.

\section{Protocol:}

This study is performed as a preliminary evaluation of the control strategy, prior to clinical trials with patients suffering from SCI. Thus an intermediate pause must be assumed in between the trials to ensure the active participation of the user, comfort in walking and to avoid muscle fatigue. In case of SCI patients, therapy procedures consider a pause of 1 minute at the end of every 2-3 minutes of walking, considering their fatigue. Since this study involves healthy subjects the experimental time can be higher and the pause time can be high in a 2:1 ratio. Hence an intermediate pause time of $2-3$ minutes is considered at the end of every 6 minutes walking test. This walking experiment is performed for 20 minutes, i.e. 3 sets of 6 minutes walking test.

The number of trials to be performed is evaluated using the probabilistic method ' $z$-test', by comparing the assistive and non-assistive method results. A maximum sample power of 0.9211 was obtained for $n=30$ trials. In this work, each gait cycle is considered as a trial and each subject performed 30 trials at the end of a 6 minutes walking test. This accounts to 90 trials/subject at the end of 20 minutes walking experiment.

\section{RESUlTS AND DisCUSSIONS}

The proposed adaptive control strategy using the humanorthosis interaction torques has been tested and evaluated with five healthy individuals of the age group $30 \pm 5$, weight $80 \pm 8 \mathrm{~kg}$, and height $1.75 \pm 0.05 \mathrm{~m}$.

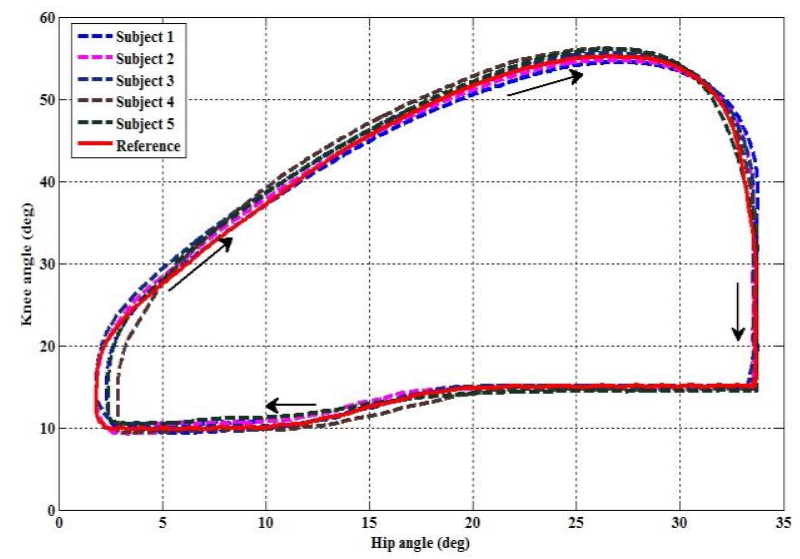

Figure 4. Reference gait pattern and the resulting mean gait pattern of each subject: Hip (deg) - Knee (deg)

The reference gait pattern and the resulting mean gait cycles of the subjects are presented in figures 4 and 5 . The subjects performed a free normal walking movement, low stiffness, with no restrictions on angle positions. The deviation from the desired trajectory was found to be high in case of the free walking. After a series of trials (10) this error decreased gradually due to the effect of the stiffness acting on the joints. The stiffness variation helped to maintain this error within a specified range and following a similar pattern of incrementing and decrementing $\mathrm{K}$ at every joint. This stiffness variation of the joint results in exerting an assistive or resistive behavior, based on the direction of the movement.

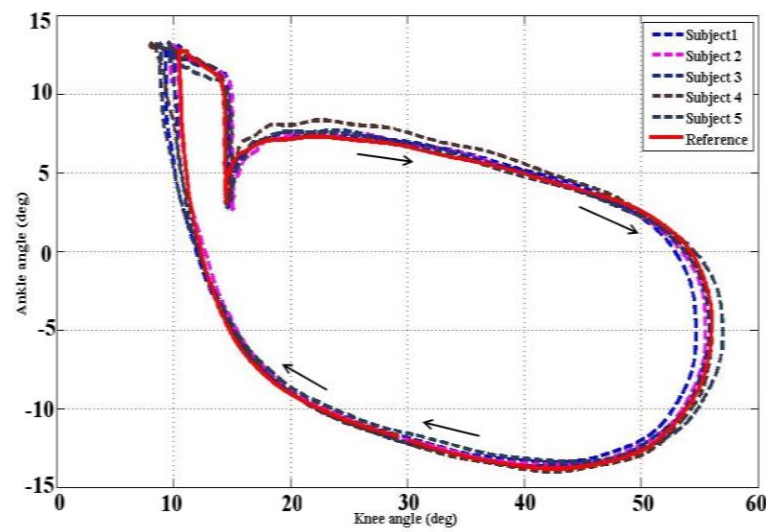

Figure 5.Reference gait pattern and mean gait pattern of each subject: Knee (deg) - Ankle (deg)

The results of one of the subjects are used to show the response of the control strategy. The gait performance of a healthy user, as shown in figures 6 and 7, demonstrates the influence of the stiffness variation proposed in this work. The initial walking with low stiffness value is presented as the 'no-assistance' mode. In comparison with the reference 
pattern, the no assisted walking is found to produce a maximum deviation. After the application of a variable stiffness, the user is able to walk within a predefined error limits. The stiffness variation also converges with respect to the movement at the end of 10 trials. At the end of 20 trials, the user is following a movement which is quite similar to the reference pattern.

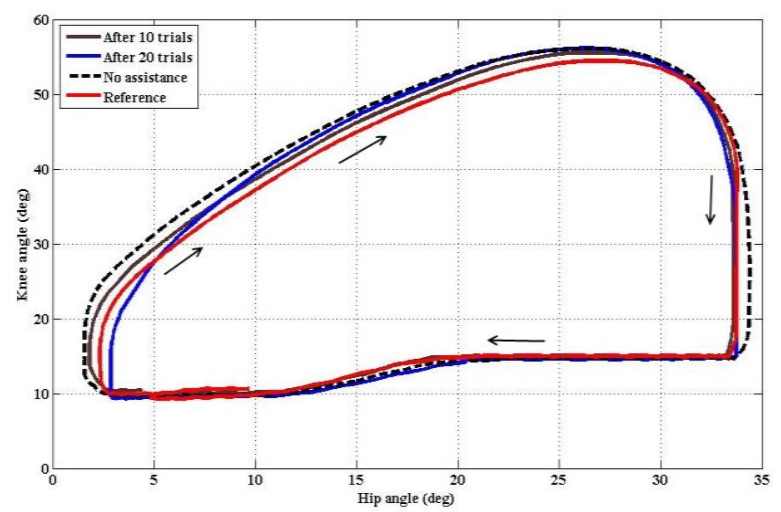

Figure 6. Changes in gait pattern of a healthy subject due to the effect of stiffness: Hip (deg)-Knee (deg)

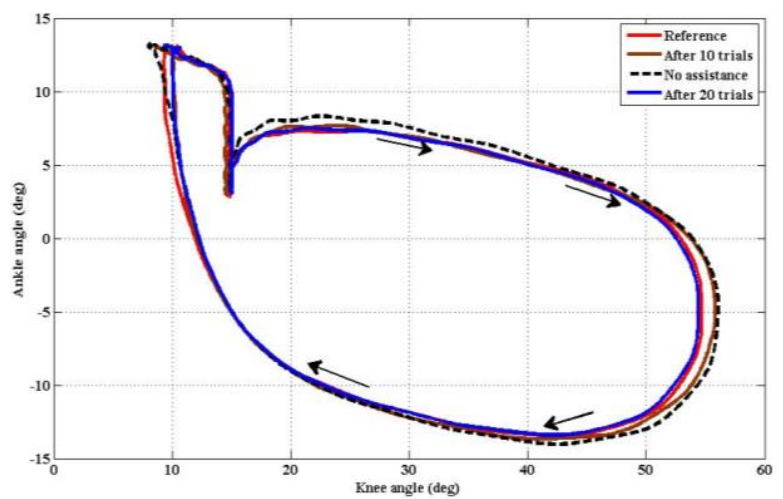

Figure 7. Changes in gait pattern of a healthy subject due to the effect of stiffness: Knee (deg)-Ankle (deg)

The error was found to be within the defined limits for all the users. The hip joint showed a little variation and more adaptable behavior in terms of stiffness changes in real time. Since the exoskeleton is a planar robot, the lateral hip

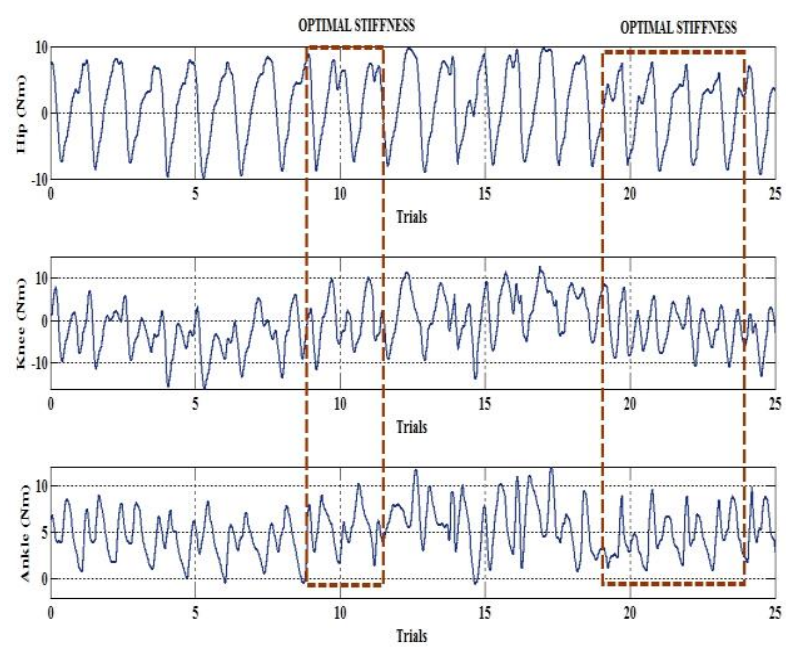

Figure 8. Interaction torques of each joint showing the change in behavior while stiffness converges to an optimal value movement cannot be monitored. However, this orthosis limitation does not affect the proposed control strategy. A significant variation of the stiffness is found in both ankle and knee joints. The hip joint stiffness varied in a short range which is evident from the interaction torques in fig 8 . This can be due to the lateral movement of the user's hip joint which compensates the joint trajectory. The interaction torque of the ankle joint is in the limits of $12 \mathrm{Nm}$ to $-3 \mathrm{Nm}$, as shown in figure 8 , and with the application of confidence factor the threshold is limited to $10 \mathrm{Nm}$ to $-1 \mathrm{Nm}$. This threshold limit is used to initiate the stiffness increment when the position error threshold is reached. Similarly in the knee joint, the interaction torques are in the limit of $14 \mathrm{Nm}$ to -14 $\mathrm{Nm}$ and after the application of the confidence factor the threshold is limited between $12 \mathrm{Nm}$ and $-12 \mathrm{Nm}$. The interaction torques are bounded within the limits even in the presence of maximum stiffness.

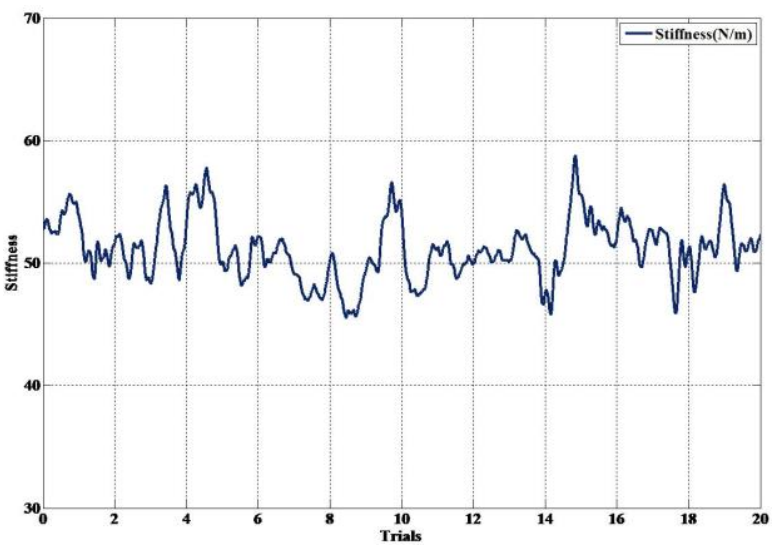

Figure 9. Stiffness $(\mathrm{N} / \mathrm{m})$ variation in the knee joint

The knee joint's flexion and extension movement plays an essential role in walking by maintaining the time instants in the transition between gait phases. Thus the stiffness variation for the knee joint was observed to converge, because of the repetitive movements, after a few gait trials, as shown in fig 9. Fig 10 shows that in knee joint, the trajectory deviation is maintained within a small range, but with a delay in the movement.

In case of the ankle joint the stiffness behavior was observed to follow a different pattern, as shown in fig 11 . This stiffness behavior is due to the compensation of ground

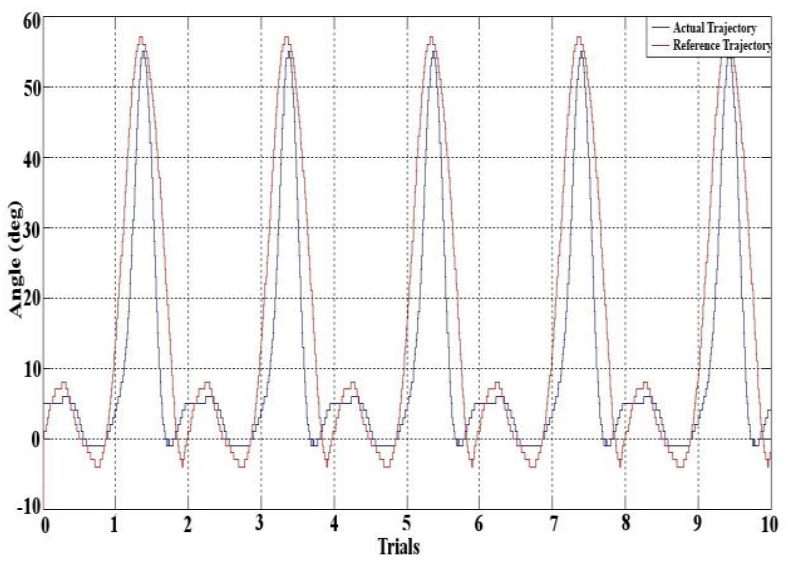

Figure 10. Knee joint trajectory (deg) of a healthy subject (redreference and blue- actual trajectory) 
reaction forces acting on the body. The stiffness value changes with respect to the trajectory deviation and the trend of the interaction torques are shown in fig 12. In the ankle joint, the deviation from the reference position is found to be higher, which explains the pattern of stiffness variation. The error in position of the joint in combination with the change in interaction torques results in a high stiffness value.

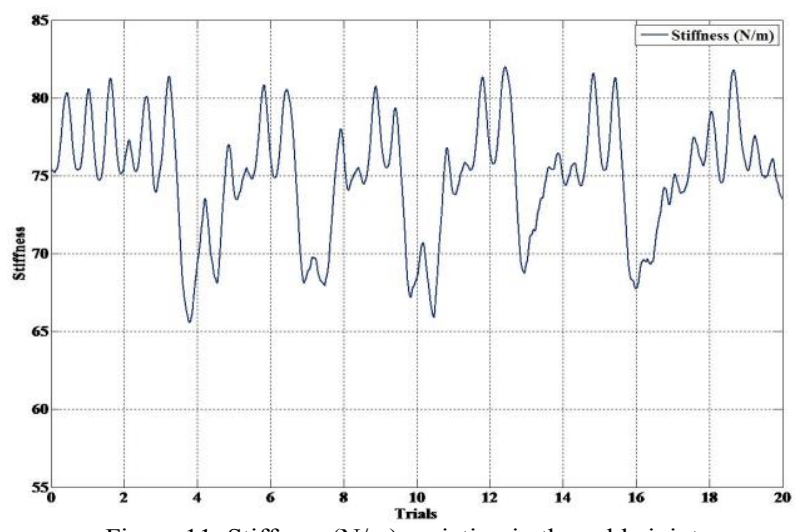

Figure 11. Stiffness $(\mathrm{N} / \mathrm{m})$ variation in the ankle joint

The confidence factor is used to act on the joint stiffness gradually. The consequence is the relax intervals that appear as negative slope (decreasing stiffness), which results in achieving a smooth behavior of the system. Lower confidence factors will result in few and shorter steps of stiffness variation, so the increment will be faster. On the contrary, a higher confidence factor will limit the increase of stiffness. The gradual increase in the stiffness value is due to the permanent difference in position error. The error in position of the joint in combination with the change in interaction torques results in a high stiffness value.

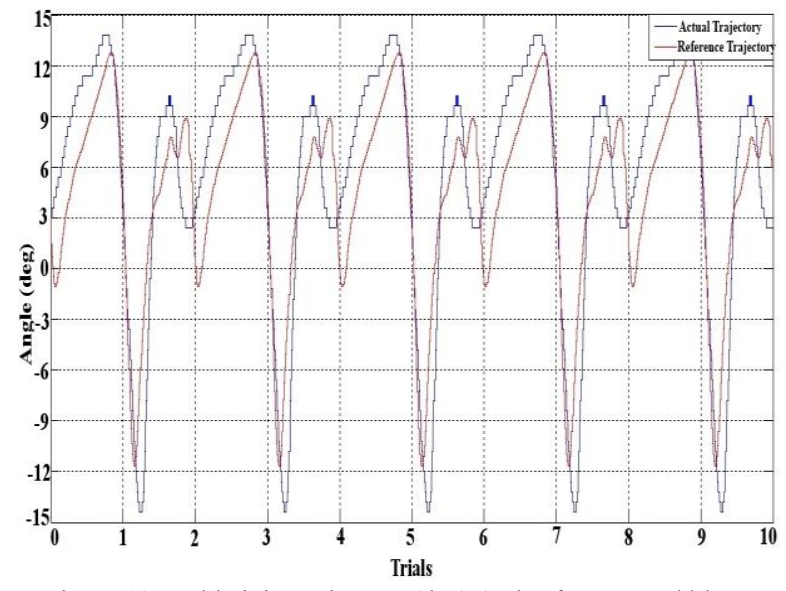

Figure 12. Ankle joint trajectory (deg) (red-reference and blueactual trajectory)

\section{CONCLUSION}

An assistive strategy has been evaluated based on position error and the human-orthosis interaction torques acting on the system, which ensures a safe and comfortable therapy. The stiffness value, for any joint, adapts dynamically to the user needs and keeps the position error bounded in the specified limits in real time. The wearable robot was tested with no body weight compensation which demonstrates the reliability of the control strategy in terms of ensuring dynamic stability in presence of ground reaction torques. The results of the proposed method were verified in comparison with a predefined gait trajectory. The experimental results showed that the evolution of the stiffness value cannot follow a similar pattern for all the joints. Similarly the stiffness value converges to a value within a given range after a series of trials. The stiffness variation was in coordination with the flexion and extension movements. This demonstrates the efficiency of the proposed method for a real time process involving multiple joints.

Future work will focus on evaluating the performance of this control in the assistance of Spinal cord injury and Stroke patients, which will include the presence of muscle stimulation (FES), acting as external disturbances for the proposed control strategy. Muscle stimulation will evidently induce higher and lower interaction torques and the system must be stable and adaptable in both cases.

\section{BIBLIOGRAPHY}

1. L. Marchal-Crespo and D. Reinkensmeyer, "Review of control strategies for robotic movement training after neurologic injury," Journal of NeuroEngineering and Rehabilitation, vol. 6, no. 20, 2009.

2. T. Yan, M. Cempini, C. M. Oddo and N. Vitiello, "Review of assistive strategies in powered lower-limb orthoses and exoskeletons," Robotics and Autonomous Systems, vol. 64, pp. 120-136, 2015.

3. R. Riener, L. Lunenburger and G. Colombo, "Human centered robotics applied to gait training and assessment," Journal of Rehabilitation Research and Development, vol. 43, no. 5, pp. 679694, 2006.

4. J. F. Veneman, R. Kruidhof, E. E. G. Hekman, R. Ekkelenkamp, E. H. F. Van Asseldonk and H. van der Kooji, "Design and evaluation of the LOPES Exoskeleton robot for Interactive gait rehabilitation," IEEE Transactions on Neural Systems and Rehabilitation Engineering, vol. 15, no. 3, pp. 379-386, 2007.

5. T. Hayashi, H. Kawamoto and Y. Sankai, "Control method of robot suit HAL working as operator's muscle using biological and dynamical information," in IEEE/RSJ International conference on Intelligent Robots and Systems (IROS), pp. 3063-3068, 2005.

6. H. Kazerooni, J. L. Racine, L. Huang and R. Steger, "On the control of the Berkeley lower extremity exoskeleton (BLEEX)," in IEEE International Conference on Robotics and Automation, ICRA, pp. 4353- 4360, 2005.

7. K. A. Strausser and H. Kazerooni, "The development and testing of a human machine interface for a mobile medical exoskeleton," in IEEE/RSJ International Conference on Intelligent Robots and Systems (IROS), pp.4911-4916, 2011.

8. M. Talaty, A. Esquenazi and J. E. Briceño, "Differentiating ability in users of the ReWalk powered exoskeleton: An Analysis of walking kinematics," in IEEE International Conference on Rehabilitation Robotics (ICORR), pp. 1-5, 2013.

9. S. Jezernik, G. Colombo and M. Morari, "Automatic gait-pattern adaptation algorithms for rehabilitation with a 4-dof robotic orthosis," IEEE Transactions on Robotics and Automation, vol. 20, no. 3, pp. 574-582, June 2004.

10. M. Bortole, A. J. Del-Ama, E. Rocon, J. C. Moreno, F. Brunetti and J. L. Pons, "A Robotic Exoskeleton for Overground Gait Rehabilitation," in IEEE International Conference on Robotics and Automation, pp. 3356-3361, 2013.

11. V.Rajasekaran, J.Aranda, A.Casals and J. L. Pons, "An Adaptive control strategy for Postural stability using a Wearable robot, " Robotics and Autonomous Systems, online version, 2014. 